\title{
High resolution spectroscopy of brown dwarfs in Taurus Detection of accretion in KPNO-Tau-3
}

\author{
D. Barrado y Navascués
}

\author{
Laboratorio de Astrofísica Espacial y Física Fundamental, INTA, PO Box 50727, 2808 Madrid, Spain \\ e-mail: barrado@laeff.esa.es
}

Received 3 February 2004 / Accepted 11 March 2004

\begin{abstract}
We present high resolution optical spectroscopy of three candidate members of the Taurus-Auriga star forming region. Based on the spectral type, the strength, profile and width of the $\mathrm{H} \alpha$ line, the detected lithium at $6708 \AA$, the location of these objects in the H-R diagram and the comparison with similar objects belonging to young stellar associations, we determine that they are bona fide members of the SFR with ages of $\sim 3 \mathrm{Myr}$, and have masses at or below the substellar limit. At least in one case, there is active accretion from a circum(sub)stellar disk. This result suggests that high mass brown dwarfs go through a classical T Tauri phase and form, as stars do, from the collapse and fragmentation of a molecular cloud.
\end{abstract}

Key words. open clusters and associations: individual: Taurus - stars: brown dwarfs

\section{Introduction}

Brown dwarfs, objects unable to fuse hydrogen in a stable manner (i.e., with masses below $\sim 0.072 M_{\odot}$, Baraffe et al. 1998), pose an important problem to the theory of stellar formation. Several formation mechanisms have been proposed, including formation like a star (from collapse and fragmentation of a molecular cloud, Padoan \& Nordlund 2004), like a planet (from a circumstellar disk) or as stellar "embryos", ejected from multiple systems before they have been able to accrete enough matter (Reipurth \& Clarke 2001; Bate et al. 2002). These proposed mechanisms have different implications for their formation, their evolution and their properties. In particular, if brown dwarfs are created like stars, they should go through a phase of active accretion from a circum(sub)stellar disk, as low mass stars do (Shu et al. 1987). On recent years, different groups have presented indirect and direct evidence of active accretion in a handful of young brown dwarfs belonging to several nearby star forming regions (SFR), open clusters and moving groups (Fernández \& Comerón 2001; Muench et al. 2001; Natta \& Testi 2001; Natta et al. 2001, 2002; Testi et al. 2002; Jayawardhana et al. 2002a,b, 2003a,b; Muzerolle et al. 2003; Barrado y Navascués et al. 2002, 2003, 2004a; Barrado y Navascués \& Martín 2003; Mohanty \& Basri 2003; Mohanty et al. 2003; Comerón et al. 2003).

In this paper, we enlarge the sample by collecting high resolution spectroscopy of three proposed members of the TaurusAuriga SFR, located at $140 \mathrm{pc}$ and about 1-3 Myr old (for an update on Taurus, see Luhman et al. 2003, and references therein).

\section{Observations}

Our Taurus targets were selected from Briceño et al. (2002). In that paper, a new sample of nine mid- to late-M candidate members were presented, based on $I c$ and $z$ ' photometry (plus 2MASS near-IR data) and low resolution spectroscopy. We have collected high resolution spectra for three of these the high mass brown dwarfs, whose spectral type is between M 5.75 and M 7.5 - with the Magellan I $6.5 \mathrm{~m}$ telescope and the MIKE echelle spectrograph on 2002, Dec. 11-14th. Although three is a small number, they represent a significant fraction of the high mass brown dwarfs belonging to this association discovered so far (about 12 in the quoted spectral range). Therefore, they can yield some information about their formation mechanism.

Additional details about the observations can be found in Barrado y Navascués et al. (2004b), where we analyze a sample of very low mass stars and brown dwarfs belonging to the $\sim 5 \mathrm{Myr}$ cluster associated to the $\lambda$ Orionis star. To optimize the signal-to-noise, we binned the data during the read-out to two by two pixels in the spatial and the spectral direction, respectively, yielding a spectral resolution of $R=25000(\sim 0.25 \AA)$, with a 0.75 arcsec slit. The spectral wavelength range of our spectra is $4500-7250 \AA$.

\section{Analysis and discussion}

\subsection{Spectral types}

Spectral types were derived by comparison with several spectral templates, using the order around $7050 \AA$, corresponding to a TiO band, following Mohanty et al. (2004), since this range is 


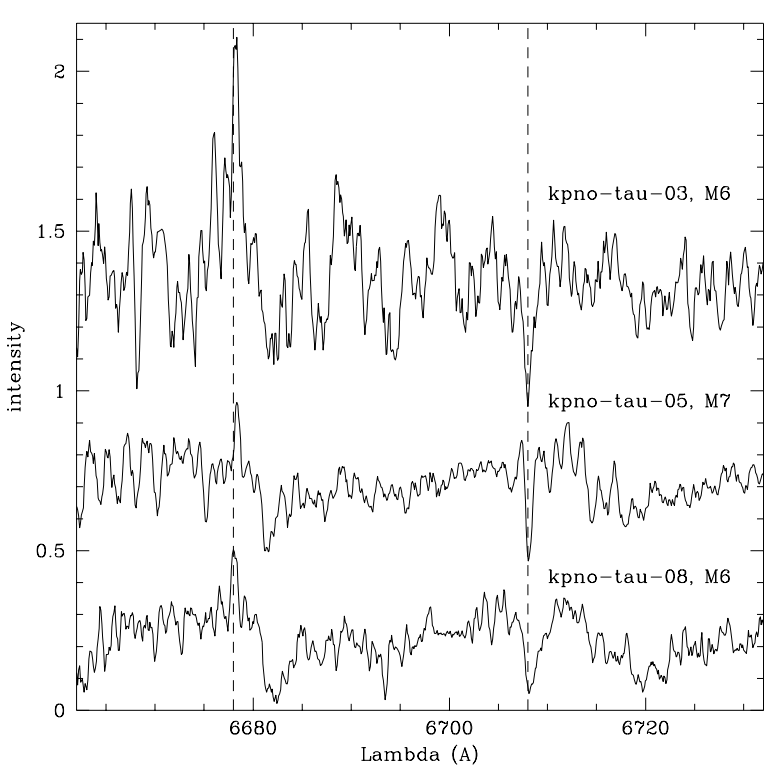

Fig. 1. Spectra around HeI $6678 \AA ̊$ and LiI $6707.8 \AA$.

very sensitive to effective temperature. Errors are estimated as half a subclass. Our values are very close to those obtained by Briceño et al. (2002) from low resolution spectra (see Table 1).

\subsection{On the lithium abundance, age, and mass}

We have detected lithium $6707.8 \AA$ in two out of the three targets (KPNO-Tau-5 and KPNO-Tau-8, Fig. 1). The spectrum of KPNO-Tau-3 has worse quality. With some caveats, the visual inspection indicates that the lithium feature is also present. Note that Briceño et al. (2002) state that they detected lithium in their low resolution spectrum (at higher signal-to-noise ratio). This element is easily destroyed in the stellar interior, as its surface abundance dependent on mass and age (as well as other second order parameters). In fact, brown dwarfs that are more massive than about $0.060 M_{\odot}$ do deplete lithium on a time scale of few tens of million years (D'Antona \& Mazzitelli 1994; Burrows et al. 1997; Baraffe et al. 1998). The Taurus-Auriga complex has an age between 1 and 3 Myr. Since our three candidate members have spectral types between M 6 and M 7 , they should have masses equal to or larger than $0.06 M_{\odot}$, according to Baraffe et al. (1998). Therefore, the detection of lithium clearly indicates that these objects are in the pre-Main Sequence (PMS) stage. Moreover, from the statistical point of view, the likelihood of having three late-M PMS interlopers whose spectral and photometric properties coincide with the Taurus sequence is negligible. Therefore, we have to conclude that they indeed belong to the association.

Figure 2 displays values of the lithium equivalent width $(W)$ measured for our targets (large asterisks), PMS members of the $\sim 5$ Myr Sigma Orionis cluster (Zapatero Osorio et al. 2002, solid squares), and stellar and substellar members of IC2391 -53 Myr- and the Pleiades -125 Myrfrom Barrado y Navascués et al. (1999, 2004b), Soderblom et al. (1993), García-López et al. (1994), Jones et al. (1996), Stauffer et al. (1998) and Jeffries et al. (1999). The solid line describe the maximum $W(\mathrm{Li})$ measured in cluster stars.

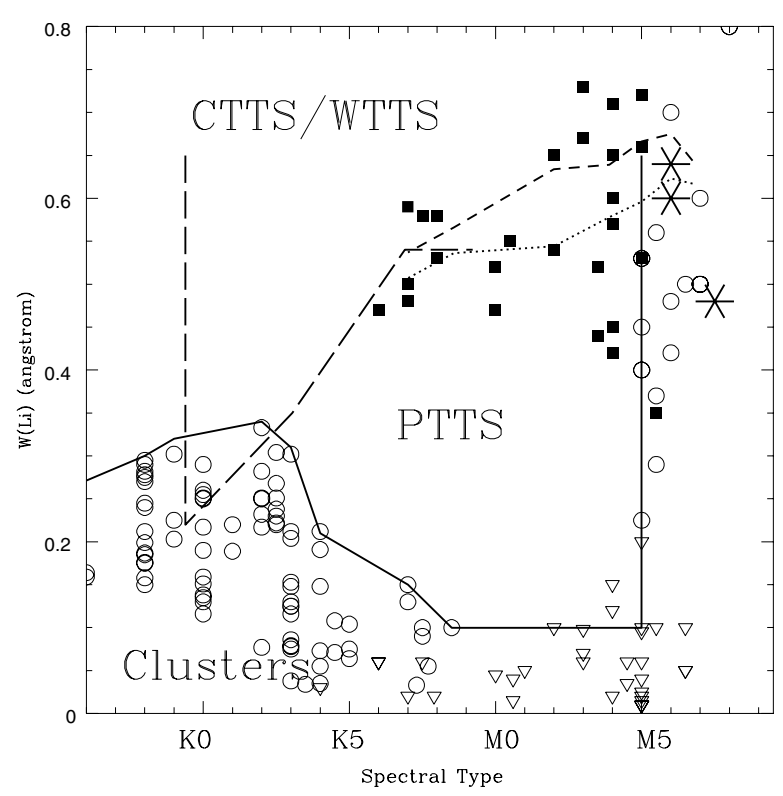

Fig. 2. Lithium equivalent width versus spectral type. The solid line corresponds to the upper envelope of the values measured in young open clusters. The long-dashed line delimits the areas for weak-line and post-T Tauri stars (adapted from Martín 1997 and Martín \& Magazzù 1999). Short-dashed and dotted lines correspond to the cosmic abundances $-A(\mathrm{Li})=3.1-$ from gravities of $\log g=4.5$ and 4.0, respectively (curves of growth from Zapatero Osorio et al. 2002). All Pleiades and IC 2391 members with measured lithium equivalent width are shown as open circles; the triangles denote upper limits. Note the lithium depletion boundary at $\sim$ M 5.5. Sigma Orionis low mass stars and brown dwarfs appear as solid squares (Zapatero Osorio et al. 2002). The big asterisks represent KPNO-Tau-3, 5 and 8.

The long-dashed curve is an update version of the criterion used by Martín (1997) and Martín \& Magazzù (1999) to distinguish between classical/weak-line T Tauri stars (CTTS and WTTs, above) and post- T Tauri stars (PTTS, below). Finally, the dotted and short dashed lines correspond to the Zapatero Osorio et al. (2002) curves of growth for $\log g=4.0$ and 4.5 and an abundance of $A(\mathrm{Li})=3.1$ (i.e., cosmic abundance). In the figure we have labeled different sections. The $W(\mathrm{Li})$ for KPNO-Tau-5 is lower than the measured values for the other two Taurus objects. This might be due to the presence of optical veiling, about $r(6700) \sim 0.2$, although we do not think this is the case. On the one hand, no other sign of accretion has been found in this object (see next section). Moreover, this equivalent width is compatible with the dispersion found in Sigma Orionis and the -undepleted- values characteristic of cluster brown dwarfs. For the other two objects, no veiling seems to be present either. If the lithium equivalent width dispersion is real both in the Taurus SFR and the Sigma Orionis cluster (the same effect is present in the Lambda Orionis association, Barrado y Navascués et al. 2004b), it might imply differences in the surface abundances due to mixing mechanisms in the stellar and substellar interior, other than pure convection (such as those proposed in solar mass stars), differences in the structure due to different stellar and substellar properties (such as fast rotation in the case of PMS stars, Martín \& Claret 1996), or to induced effects related to activity and/or rotation on the spectral 
Table 1. Data for the three Taurus low mass members.

\begin{tabular}{cccc}
\hline \hline & \multicolumn{3}{c}{ KPNO-Tau } \\
\cline { 2 - 4 } & $\# 3$ & $\# 5$ & $\# 8$ \\
\hline$I c$ & 15.77 & 15.08 & 15.10 \\
$J$ & $13.323 \pm 0.022$ & $12.640 \pm 0.024$ & $12.946 \pm 0.022$ \\
$H$ & $12.501 \pm 0.022$ & $11.918 \pm 0.024$ & $12.367 \pm 0.021$ \\
$K s$ & $12.079 \pm 0.021$ & $11.536 \pm 0.018$ & $11.985 \pm 0.022$ \\
Sp. type & $\mathrm{M} 6(\mathrm{M} 6)$ & $\mathrm{M} 7(\mathrm{M} 7.5)$ & $\mathrm{M} 6(\mathrm{M} 5.75)$ \\
Width H $\alpha\left(\mathrm{km} \mathrm{s}^{-1}\right)$ & 310 & 125 & $240^{1}$ \\
$W(\mathrm{H} \alpha)(\AA)$ & $81.4 \pm 5.7(130)$ & $21.1 \pm 0.9(30)$ & $12.0 \pm 1.0(17.5)$ \\
$W(\mathrm{HeI} 6678)(\AA)$ & $3.5:(6.5)$ & $0.2:$ & $0.2:$ \\
$W(\mathrm{Li})(\AA)$ & $0.60($ Yes $)$ & 0.48 & 0.78 \\
$\log \mathrm{Lum}(\mathrm{bol}) / L_{\odot}$ & $-1.66 /-1.76^{2}$ & $-1.70 /-1.66^{2}$ & $-1.65 /-1.76^{2}$ \\
$\operatorname{Mass}\left(M_{\odot}\right)$ & $0.07^{3}$ & 0.06 & $0.07^{3}$ \\
$\log L(\mathrm{H} \alpha) / L(\mathrm{bol})$ & -2.87 & -3.70 & -3.70 \\
$T_{\text {eff }}(\mathrm{Luhman} 1999)$ & 2990 & 2890 & 2990 \\
\hline
\end{tabular}

Values in parenthesis are from low resolution spectroscopy by Briceño et al. (2002). A double colon implies a large uncertainty in the measurement.

${ }^{1} 180 \mathrm{~km} \mathrm{~s}^{-1}$ without the asymmetry.

${ }^{2}$ From $I c$ and $K s$, respectively.

${ }^{3} 0.08 M_{\odot}$ from the HR diagram. The listed values are from $I c$ and Baraffe et al. (1998)

feature itself or the surrounding continuum (see the discusion in Barrado y Navascués et al. 2001 and references therein).

As a summary, based on these data, mainly from the detection of lithium and accretion when present (see next section), we can conclude that these three objects are young and belong to the Taurus region. In the case of KPNO-Tau-5, due to its spectral type (M7) its substellar nature is well established. The other two are located at the substellar borderline and their nature is not so firmly established due to uncertainties in the models and spectral type determination.

Once membership of the stellar association had been proved, we derived the bolometric magnitudes from the following: $I c$ and $K s$, the distance modulus $(m-M)_{0}=5.731(140 \mathrm{pc}$, Kenyon et al. 1994), the reddening derived by Briceño et al. (2002) and the bolometric corrections by Comerón et al. (2000) and Tinney et al. (1993) for these two bands ( $I c$ and $K s$, respectively). Masses were computed based on models by Baraffe et al. (1998). Effective temperatures were obtained using several scales, namely Luhman (1999) for intermediate gravity and Leggett (2000, 2001). All the measured and derived values are listed in Table 1.

Figure 3 displays the HR diagram. The isochrones and evolutionary tracks - solid and dashed lines, respectively-are from Baraffe et al. (1998). Asterisks and open stars correspond to bolometric luminosities obtained from $I c$ and $K s$, respectively. Note that the plotted values for KPNO-Tau-3 and KPNO-Tau8 have been offset by an arbitrary small shift, as they practically coincide. For this particular diagram, we made use of the effective temperature scale by Luhman (1999). Other temperature scales, such as that from Leggett et al. (2001), would shift the location of these three objects to the right hand-side, making them younger and less massive. Note, however, that Luhman' scale has been tuned specifically for the model we are using here. In any case, irrespectively of the selection of models (alternative models are, for instance, Burrows et al. 1997;

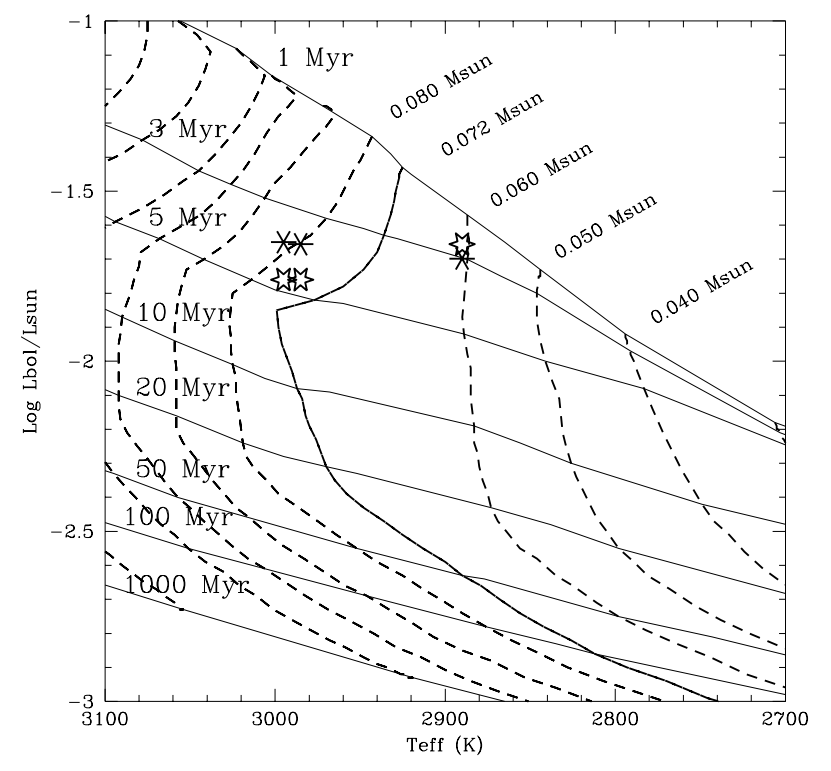

Fig. 3. HR diagram of KPNO-Tau-3 and 8 (on top of each other) and KPNO-Tau-5. The values represented by asterisks and open stars were derived from $I c$ and $K s$ magnitudes, respectively.

D'Antona \& Mazzitelli 1994, 1997, 1998; Chabrier et al. 2000; Baraffe et al. 2002), bolometric corrections and effective temperature scale, these members are at or below the substellar frontier and have an age of about $3 \mathrm{Myr}$.

\subsection{Near infrared photometry, $\mathrm{H} \alpha$ emission and accretion}

None of our three targets seems to show either forbidden lines, characteristics of outflows, or near infrared excess, coming from an accretion disk. Figure 4 compares the colors $(I c-J)$ and $(H-K s)$, and includes the Main Sequence locus 


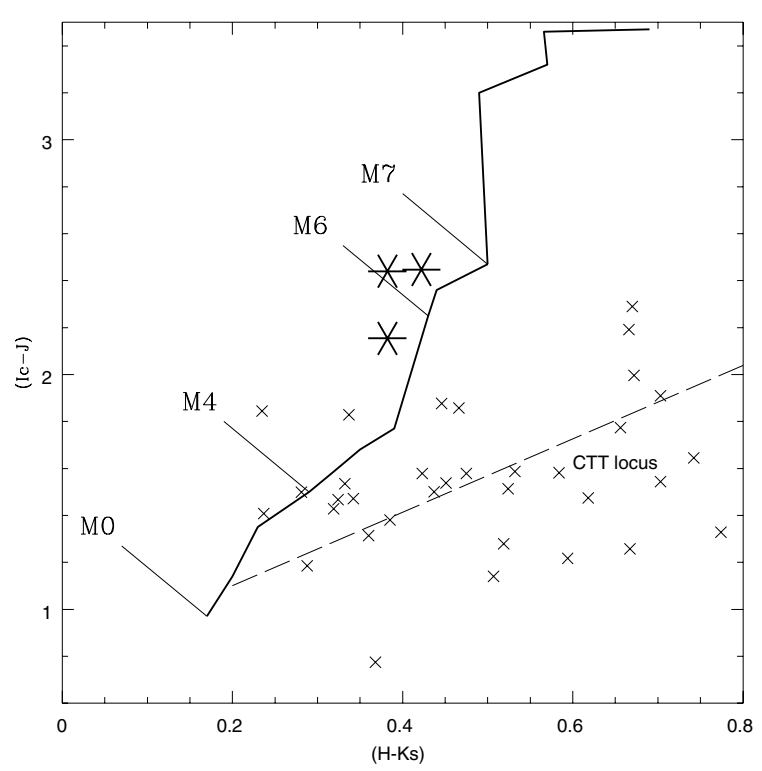

Fig. 4. Color-color diagram. The position of the Taurus BD candidates are indicated with large asterisks. Crosses indicate the position of classical T Tauri stars belonging to the Orion stellar population (Herbig \& Bell 1988). The thick-solid and dashed lines correspond to the loci of the main sequence stars (from Bessell \& Brett 1988; Kirkpatrick et al. 2000; Leggett et al. 2001) and CTT stars (Meyer et al. 1997; Barrado y Navascués et al. 2003), respectively.

(Bessell \& Brett 1988; Kirkpatrick et al. 2000; Leggett et al. 2001), the classical T Tauri stars locus (Meyer et al. 1997; Barrado y Navascués et al. 2003) and classical T Tauri stars from Orion (Herbig \& Bell 1988). As can be seen, the Taurus objects have photometric properties (data from 2MASS) similar to those of MS stars of similar spectral type and, as stated above, no near-IR excess is seen. This by itself, does not prove or disprove the presence of a circum(sub)stellar disk, since a hole can be present or the disk temperature can be very cold. Moreover, the IR excess depends on the orientation of the disk. See, for example, the case of LS-RCrA 1, an M 6.5 brown dwarf in the RCrA dark cloud (Barrado y Navascués et al. 2004a). Additional observations at longer wavelengths, more sensitive to cooler disks, both from ground-based telescopes and space-borne instruments such as those in the Spitzer Space Telescope, can help to shed some light in this issue.

We have detected and measured $\mathrm{H} \alpha$ in emission for these three objects. The profiles of this feature are displayed in Fig. 5. Note the possible asymmetry in KPNO-Tau- 8 , the double peak in KPNO-Tau-5 and KPNO-Tau-3, and the width of the line $\left(310 \mathrm{~km} \mathrm{~s}^{-1}\right)$ in the last object, typical of accreting brown dwarfs (White \& Basri 2003; Jayawardhana et al. 2003a). In fact, the $W(\mathrm{H} \alpha)$ of KPNO-Tau-3 is above the criterion defined by Barrado y Navascués \& Martín (2003) which discriminates between accreting and non-accreting objects, as Fig. 6 clearly indicates. We note, however, that this criterion depends on low resolution spectroscopy, which normally yields larger equivalent widths than higher resolution data. In this diagram, we have included classical and weak-line $\mathrm{T}$ Tauri stars belonging to Taurus as solid and open circles. Members without classification are included as crosses. Our three targets appear as large

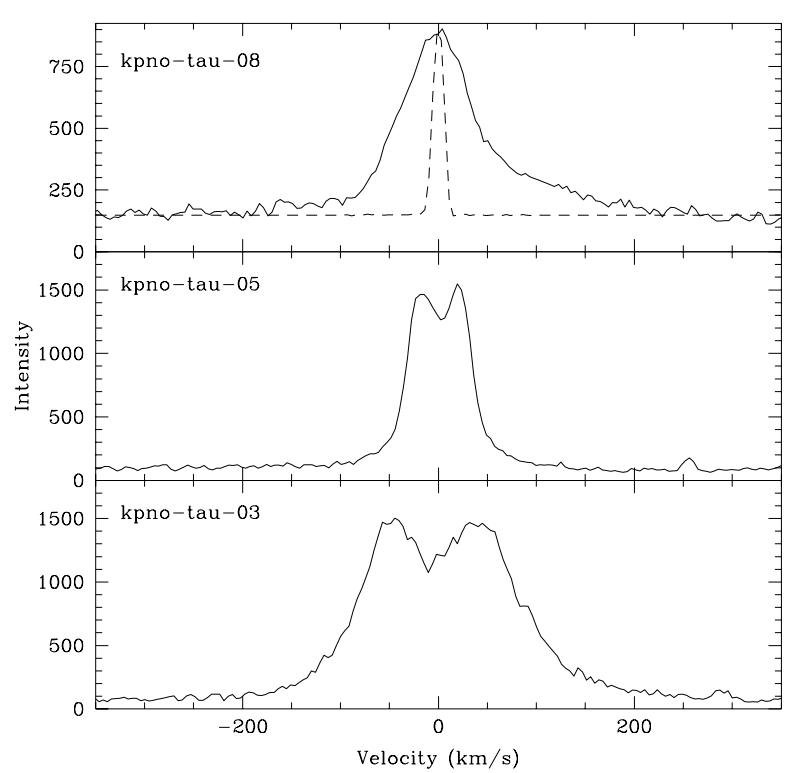

Fig. 5. H $\alpha$ profiles. The instrumental profile is included as a dashed line (top spectrum).

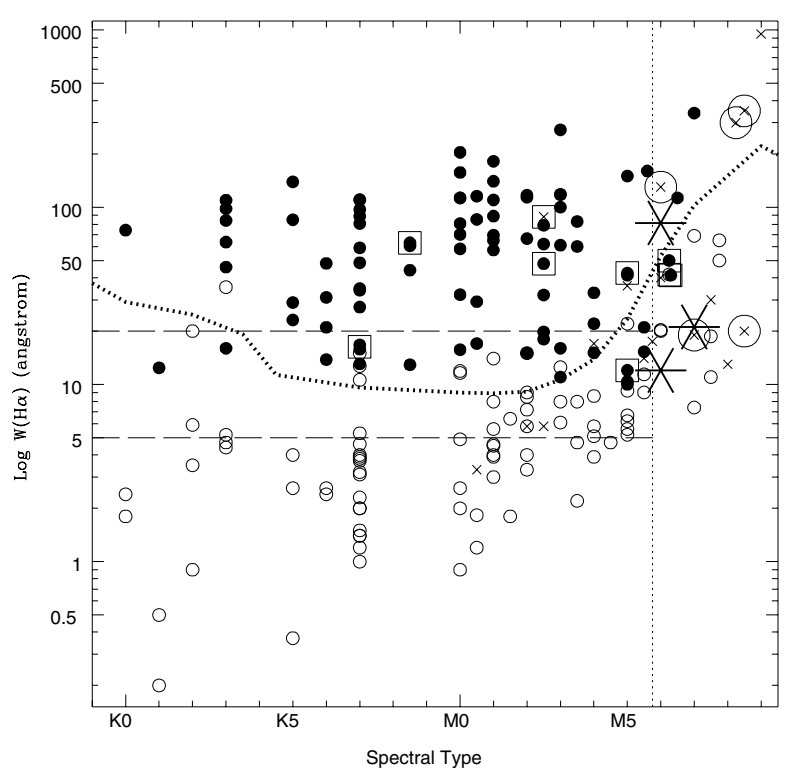

Fig. 6. H $\alpha$ equivalent widths for members of the Taurus SFR. Solid and open circles correspond to classical and weak-line T Tauri stars. Objects with no classification are shown as crosses. Large open circles or square represent objects with mid-IR excesses and forbidden lines, respectively. The three objects studied here are displayed as large asterisks. The dotted, bold curve is the saturation criterion, whereas two previously proposed criteria (5 and $20 \AA$ ) to separate CTTS and WTTS are included as long-dashed, thin horizontal segments. The vertical dotted segment denotes the location of the substellar frontier.

asterisks. Overlapping squares and big circles denote those members with forbidden emission lines and near-infrared excesses, respectively. The large $W(\mathrm{H} \alpha)$ of KPNO-Tau-3 agrees with the fact that we also detect HeI $6678 \AA$, another accretor indicator (see Fig. 1).

Active accretion in at least one Taurus member whose mass is close or below the substellar limit is important for several reasons. First, although the sample studied in this paper is very 
small, it suggests that a significant fraction of the very low mass stars and high mass brown dwarfs might harbor an accretion disk. Second, the new data amass additional evidence for accretion in the substellar domain. To the best of our knowledge, there are only three papers dealing with high resolution spectra in Taurus brown dwarfs (White \& Basri 2003; Muzerolle et al. 2003; Jayawardhana et al. 2003a). The first work found three accretors in a sample of ten very low mass stars and brown dwarfs, although the less massive, a M6.5 (GM Tau), has a mass just above the substellar limit. The second paper includes four objects whose spectral type is M6 or M7. None of them seem to undergo active accretion based on the width of $\mathrm{H} \alpha$, although the authors claim that one of them, namely MHO-5, is accreting based on the detection of forbidden lines of oxygen at 6300 and $6363 \AA$ and CaII IRT. Moreover, three out of the four were observed at moderate resolution, including MHO-5 $(R \sim 8000)$. Regarding the study by Jayawardhana et al. (2003a), the four M 7-M 7.75 brown dwarfs discussed there, with masses down to $0.05 M_{\odot}$ (again, using Baraffe et al. 1998 models), do not show accretion either. Muzerolle et al. (2003) also analyzed seven Taurus members whose spectral types are M 4.75-M 5.75 (just above or at the substellar borderline). Three out of these seven are accreting, based on the width of $\mathrm{H} \alpha$ and other spectral indicators. Therefore, up to now, our study presents the only brown dwarf belonging to Taurus (KPNO-Tau-03) at the substellar limit which has been proved to be accreting.

In combination with the studies quoted in the previous paragraph, our results indicate that about $10 \%$ of the Taurus brown dwarfs (one out of 11) is actively accreting. This fraction is much smaller than the accretion frecuency in low mass stars in the association, or than the estimate for the substellar domain (about 50\%) based purely on the strength of $\mathrm{H} \alpha$ measured in low resolution spectra (Barrado y Navascués \& Martín 2003). The statistical criterion defined in this last work is based on the saturation of the chromospheric activity, as measured in several young open clusters (namely IC 2391, Alpha Per and the Pleiades, with ages ranging from about 50 to $125 \mathrm{Myr}$ ). The discrepancy in the fraction of accreting brown dwarfs might imply that there is an additional source of flux in the $\mathrm{H} \alpha$ line or that the accreting criterion based on the $\mathrm{H} \alpha$ width at $10 \%$ of the maximum intensity, as defined by Jayawardhana et al. (2003a), i.e. $200 \mathrm{~km} \mathrm{~s}^{-1}$, is too restrictive.

The detection of accretion in substellar objects indicates that they undergo a phase similar to classical T Tauri stars (for a review, see Bertout 1989; or Appenzeller \& Mundt 1989). A handful of other brown dwarfs belonging to other young stellar associations, with ages ranging from 1 to $10 \mathrm{Myr}$, have been observed with high resolution spectroscopy and have had the presence of accretion confirmed. In at least one, LSRCrA 1 (Fernández \& Comerón 2001; Barrado y Navascués et al. 2004a), outflows have been seen, by means of the detection of intense, narrow forbidden lines. In a previous paper we have named them classical $\mathrm{T}$ Tauri substellar analogs (CTTSA). Therefore, we can conclude, at least in the case of high mass brown dwarfs (Mass $\sim 0.072-0.04 M_{\odot}$ ), that they are formed as low mass stars, by fragmentation and collapse of the original molecular cloud. Of course, additional studies are needed to confirm this preliminary conclusion, in particular high resolution imaging in the near- and mid- infrared, as well as in the optical. Narrow band imaging might show whether these objects present outflows similar to those observed in some classical T Tauri stars.

\section{Summary}

We have collected high-resolution spectroscopy for the members of the Taurus-Auriga SFR. They have M 6-M7 spectral types with moderate to intense $\mathrm{H} \alpha$ emission, with profiles that, at least in one case, can be classified as typical of an accretor. We detect lithium too, which indicates that they belong to the pre-main sequence (more strictly, the equivalent width imposes a maximum age of about $30 \mathrm{Myr}$, based on Baraffe et al. 1998). Moreover, at least one, possibly two of them, are accreting material, as shown by the $\mathrm{H} \alpha$ profile, providing an additional, stronger constrain to the age, about $10 \mathrm{Myr}$. Therefore, a minimum of $10 \%$ (one out of eleven) of the high mass brown dwarfs known in the cluster show accretion (i.e., circumsubstellar disks). Note, however, that these objects have no near-infrared excess and do not show forbidden lines, although HeI $6678 \AA$, another signpost of accretion, appears in the spectrum of one of them, and possibly also in the other two. Since the likelihood of having a stellar association younger than $30 \mathrm{Myr}$ in the same line-of-sight as Taurus is very low, we can safely conclude that these objects belong to this SFR. Based on the new spectral types and magnitudes in the optical and infrared, we derived masses around the substellar limits, and an age close to 3 Myr. Finally, the detection of accretion in the substellar domain provides another clue about the formation mechanism of high mass brown dwarfs, suggesting that they form as stars, by fragmentation and collapse of a molecular cloud.

Acknowledgements. This work has been supported by the Spanish "Programa Ramón y Cajal" and the PNAyA AYA2003-05355. Comments by M. R. Zapatero Osorio are appreciated.

\section{References}

Appenzeller, I., \& Mundt, R. 1989, A\&ARv, 1, 291

Baraffe, I., Chabrier, G., Allard, F., et al. 1998, A\&A, 337, 403

Baraffe, I., Chabrier, G., Allard, F., \& Hauschildt, P. H. 2002, A\&A, 382,563

Barrado y Navascués, D., Stauffer, J. R., \& Patten, B. M. 1999, ApJ, 522, L53

Barrado y Navascués, D., García López, R. J., Severino, G., \& Gomez, M. T. 2001, A\&A, 371, 652

Barrado y Navascués, D., Zapatero Osorio, M. R., Martín, E .L., et al. 2002, A\&A, 393, L85

Barrado y Navascués, D., \& Martín, E. L. 2003, AJ, 126, 2997

Barrado y Navascués, D., Béjar, V. J. S., Mundt, R., et al. 2003, A\&A, 404, 171

Barrado y Navascués, D., Mohanty, S., \& Jayawardhana, R. 2004a, ApJ, 604, L284

Barrado y Navascués, D., Stauffer, J. R., \& Jayawardhana, R. 2004b, ApJ, in press

Bate, M. R., Bonnell, I. A., \& Bromm, V. 2002, MNRAS, 332, L62

Bertout, C. 1989, ARA\&A, 27, 351 
Bessel, M. S., \& Brett, J. M. 1987, PASP, 100, 1134

Briceño, C., Luhman, K. L., Hartmann, L., et al. 2002, ApJ, 580, 312 Burrows, A., Marley, M., Hubbard, W. B., et al. 1997, ApJ, 491, 856

Chabrier, G., Baraffe, I., , Allard, F., \& Hauschildt, P. 2000, ApJ, 542, L119.

Comerón, F., Neuhüser, R., \& Kaas, A. A. 2000, A\&A, 359, 269

Comerón, F., Fernández, M., Baraffe, I., et al. 2003, A\&A, 406, 1001

D'Antona, F., \& Mazzitelli, I. 1994, ApJS, 90, 467

D'Antona, F., \& Mazzitelli, I. 1997, in Cool Stars in Clusters and Associations, ed. R. Pallavicini, \& G. Micela, Mem. Soc. Astron. Italiana, 68 (4), 807

D’Antona, F., \& Mazzitelli, I. 1998, in Brown Dwarfs and Extrasolar Planets, ed. R. Rebolo, E. Martín, \& M. R. Zapatero Osorio, ASP Conf. Ser., 134, 442

Fernández, M., \& Comerón, F. 2001, A\&A, 380, 264

García López, R. J., Rebolo, R., \& Martín, E. L. 1994, A\&A, 282, 518

Herbig, G. H., \& Bell, K. R. 1988, Lick Observatory Bulletin, Lick Observatory

Jayawardhana, R., Mohanty, S., \& Basri, G. 2002a, ApJ, 578, L141

Jayawardhana, R., Holland, W. S., Kalas, P., et al. 2002b, ApJ, 570, L93

Jayawardhana, R., Mohanty, S., \& Basri, G. 2003a, ApJ, 592, 282

Jayawardhana, R., Ardila, D., Stelzer, B., et al. 2003b, AJ, 126, 1515

Jeffries, R. D. 1999, MNRAS, 309, 189

Jones, B. F., Fischer, D. A., \& Stauffer, J. R. 1996, AJ, 1112, 1562

Kenyon, S. J., Dobrzycka, D., \& Hartmann, L. 1994, AJ, 108, 1872

Kirkpatrick, D., Reid, I. N., Liebert, J., et al. 2000, AJ, 120, 447

Leggett, S. K., Allard, F., Dahn, C., et al. 2000, ApJ, 535, 965

Leggett, S. K., Allard, F., Geballe, T. R., Hauschildt, P. H., \& Schweitzer, A. 2001, ApJ, 548, L908
Luhman, K. L. 1999, ApJ, 525, L466

Luhman, K. L., Briceño, C., Stauffer, et al. 2003, ApJ, 590, 348

Martín, E. L., \& Claret, A. 1996, A\&A, 306, 408

Martín, E. L. 1997, A\&A, 321, 492

Martín, E. L., \& Magazzù, A. 1999, A\&A, 342, 173

Meyer, M. R., Calvet, N., \& Hillenbrand, L. A. 1997, AJ, 114, 288

Mohanty, S., \& Basri, G. 2003, ApJ, 583, 451

Mohanty, S., Jayawardhana, R., \& Barrado y Navascués, D. 2003, ApJ, 593, L109

Mohanty, S., Basri, G., Jayawardhana, R., et al. 2004, ApJ, submitted Muench, A. A., Alves, J., Lada, C. J., \& Lada, E. A. 2001, ApJ, 558, L51

Muzerolle, J., Hillenbrand, L., Calvet, N., Briceño, C., \& Hartmann, L. 2003, ApJ, 592, 266

Natta, A., \& Testi, L. 2001, A\&A, 376, L22

Natta, A., Testi, L., Comerón, F., et al. 2002, A\&A, 393, 597

Padoan, P., \& Nordlund, Å. 2002, ApJ, 576, 870

Reipurth, B., \& Clarke, C. 2001, AJ, 122, 432

Shu, F., Adams, F., \& Lizano, S. 1987, ARA\&A, 25, 23

Soderblom, D. R., Jones, B. F., Balachandran, S., et al. 1993, AJ, 106, 1059

Stauffer, J. R., Schultz, G., \& Kirkpatrick, J. D. 1998, ApJ, 499, L199

Testi, L., Natta, A., Oliva, E., et al. 2002, ApJ, 571, 155

Tinney, C. G., Mould, J. R., \& Reid, I. N. 1993, AJ, 105, 1045

White, R. J., \& Basri, G. 2003, ApJ, 582, 2109

Zapatero Osorio, M. R., Béjar, V. J. S., Pavlenko, Ya., et al. 2002, A\&A, 384, 937 